\title{
The Holonic Production System: A Multi Agent Simulation Approach*
}

\author{
Gandolfo Dominici $^{1}$, Pierluigi Argoneto ${ }^{2}$, Paolo Renna ${ }^{2}$, Luigi Cuccia ${ }^{3}$ \\ ${ }^{1}$ S.E.A.F., University of Palermo, Faculty of Economics, Palermo, Italy; ${ }^{2}$ D.I.F.A., University of Basilicata, Faculty of Engineering, \\ Basilicata, Italy; ${ }^{3}$ D.T.M.P.I.G., University of Palermo, Faculty of Engineering, Palermo, Italy. \\ Email: gandolfodominici@unipa.it, paolo_renna@yahoo.it, \{pieroargoneto, luigi.cuccia\}@gmail.com
}

Received May $19^{\text {th }}, 2010$; revised June $3^{\text {rd }}, 2010$; accepted July $29^{\text {th }}, 2010$.

\begin{abstract}
Today's turbulent markets are facing unpredictable and sudden variations in demand. In this context, the Holonic Production System (HPS) seems to be able to overcome the operational and economic problems of traditional production systems. The HPS' ability to adapt and react to business environment changes, whilst maintaining systemic synergies and coordination, leverage on its network organizational structure, assuring both flexibility and profitability. In this paper we study HPS experimentally, modeling holon-firms as agents. In our simulation, holon-firms interact both with each other and with the external environment without predetermined hierarchies and following their own aims and internal decision rules with a negotiation-based control system. The Multi Agent System Approach we propose aims to evaluate and test the performance of the HPS to adjust to changes in market demand by simulating variations in holon-firms' capacity and reconfiguration costs in real time in a distributed enterprise network. Hence we demonstrate that, through a collaborative negotiation approach, the HPS results in a better adaptability and improved network responsiveness.
\end{abstract}

Keywords: Holonic Production System (HPS), Multi Agent System (MAS), Distributed Enterprise Network

\section{Introduction and Theoretical Framework: Why the HPS?}

Mass production proved its effectiveness in stable environments with continuous growth trends, until the end of the 1980's. Before that time, the hierarchical pattern on which mass production is founded, assumed the steadiness of social, economic and technological factors. Since the beginning of the 1990's, this pattern begun to show its weaknesses due to the increasing instability and the growing systemic complexity of business environments. The spread of Internet made it possible for firms to use a low cost, worldwide extended, informative infrastructure which brought profound changes in the market. Information and Communication Technology (ICT) allows to develop production networks between firms which can be delocalized and dispersed in space. These changes caused the shift from "mass production" to "mass-customisation". In turbulent markets, the reduced flexibility of mass production due to hierarchical control systems

*The manuscript was contributed by four authors, G. Dominici contributed Section 1; P. Argoneto contributed Sections 2, 4 and 6; P. Renna contributed Sections 2, 5 and 6; L. Cuccia contributed Sections 6 and 7. leads almost inevitably to criticalities. The problems concerned with unpredictable variations of demand rate are:

- unnecessary increases in capacity and inventory level, which reduce efficiency;

- decline of service level, resulting in a negative impact on customer satisfaction.

In order to fulfil the new needs for agility, it becomes unavoidable for firms to develop an extremely flexible production structure able to $[1,2]$ :

1) react to the market environment's turbulences;

2) survive to the changes of production system through the adoption of new technologies;

3 ) adapt to the uncertainties of production systems in such environments.

The literature on this topic shows several trends which production and supply chain systems have to adapt to [2-4]:

1) the paradigm shift from mass production to semipersonalized production;

2) the opening to collaboration with other agents so as to speed up production innovation and processes;

3 ) the decisive role of effective and efficient cooper- 
ation within networks;

4) the awareness of the problems related to the implementation of centralized control systems considering the differences of information, experiences, activities and objectives among the entities participating to the same network.

These changes call for new organizational structures. Traditional hierarchical systems show several shortfalls in these new business environments:

1) they strongly limit the reconfiguration, the reliability and the growth capacities of the organization [4];

2) their complexity grows together with the size of the organization [5];

3) communication among the elements of the system is rigorously determined ex ante and vertically limited [6];

4) the structure's modules cannot take initiatives, thus reducing the system's readiness to react therefore resulting not agile in turbulent environments [7];

5) the structure is expensive to build and to preserve.

Heterachical systems do not have the limitations of hierarchical systems, as they are able to maintain flexibility and adaptability to external stimuli. In heterachical systems, hierarchy is excluded and control is managed by the single "agents" of the system. Agents interrelate with their environment and with other agents according to their own characteristics and scopes. In this system devoid of prearranged hierarchies, control is based on negotiation [8].

In the field of artificial intelligence, the term agent is used to identify the intelligent elements of a system, who act in the environment as entities able to be conscious of situations and who pursue an aim; such agents must encompass the following attributes $[8,9]$ :

- autonomy, they act without the help or guide of any superior entity;

- social ability, they interact with other agents;

- reactivity, they perceive their environment and respond rapidly to changes;

- pro-activity, they are able to have initiative and specific behaviors for a specific scope.

Though they are agile, heterachical systems are not capable of operating according to predefined plans, for this reason their behavior is hardly predictable, thus increasing unpredictability in systemic dynamics. Heterarchical structures work well in simple, non complex and homogeneous environments with abundance of resources [7], while in complex environments with shortage of resources they can cause instability and waste. Hence it is necessary to create a system which is able to assure both performance and reactivity at the same time.

${ }^{1}$ Holistic scientific paradigm focusing on the study of Complex Adaptive Systems (C.A.S.).
Theories on living organisms and social organizations have been used as interpretive lenses to analyse business systems and firms' networks. In this view organizations are represented as living systems. The holonic paradigm emerges within this view, stemming from the holistic ${ }^{1}$ approach and the viable system approach [10]. The holonic paradigm originates from the thought of Arthur Koestler [11] that highlights how complex systems derive from the union of stable and autonomous sub-systems, which are able to survive turbulences and, at the same time, cooperate to shape a more complex system. Koestler underlines that the analysis of both the biological and the physical world shows that it is necessary to take into account the links between the whole and the part of the entities we observe. According to Koestler in order to understand complex systems, it is not enough to study atoms, molecules, cells, individuals or systems as independent entities, but it is essential to consider such unities as concurrently parts of a larger whole; in other words, we have to consider the holon. The term holon is a blend of the ancient Greek "Ö $\lambda$ o $\varsigma$ " with the meaning of "whole" and the suffix "o" $v$ " meaning entity or part; thus the whole is set up of parts which, unlike atoms, are also entities [2]. The holon is, in fact, a whole which includes, simultaneously, the elements or the sub-parts which structure it and give it a functional meaning. Holons act as intelligent, autonomous and cooperative entities which work together inside temporary hierarchies called holarchies. A holarchy is a hierarchy of self-regulating holons working, in harmony with their environment, as autonomous wholes which are hierarchically superior to their own parts and, in unison, are parts dependent on the control of superior levels. There are three pillars of holonic system [12]:

1) a shared-value system which allows the spontaneous and continuous interaction among groups of people who are geographically dispersed and are not linked by legal or ownership ties, consenting to access the economies of cooperation and increasing the stability of the system. Examples of shared value systems are some of the elements of lean production, that are often embedded in the company's vision, such as the principle of continuous improvement (kaizen);

2) a distributed network information system which is a neural sub-system [13] supporting real time supply of information between operating units. Such a system consents the pursuit of maximum income by increasing the capacity to sense and exploit new business opportunities;

3) an autonomous distributed hierarchy, which is based on the ability of each autonomous part to become leader according to the requirements of specific 
situations caused by the turbulent changes in the environment. Every entity is able to directly interact with other entities without mediation. Due to this property of holonic systems, every holon has potentially the same importance and the same responsibility; the involvement of a holon as operative unit is based on its knowledge and competencies and is not a consequence of predefined leadership (Figure 1).

As the reader can see, in the scheme proposed in Figure 1, the holons are represented as networked agents which define the production system through coordination and cooperation [2]. Holons can act as agents of three different functions: mediator, request and offer functions. The holon converts its function due to the necessity of mediation (mediator) or following its state of over (offer) or under (request) loaded of production capacity.

The mediator function is a negotiation function, which is distributed among holons. The mediator-negotiation function has a particular relevance for our simulation because it manages the distribution of resources among holons. We consider the mediator-negotiation function as a primary function and we give it a central role in our analysis.

Thanks to computer simulation methodology, it is possible to test the performance of complex systems scientifically. According to the scheme in Figure 1, we propose a simulation, based on a multi agent architecture and a negotiation protocol (which shares the capacity among the holon-firms), in order to test the proposed approach comparing it with the "central planner with full knowledge", which is here considered as benchmark.

\section{Model Formulation}

We represent firms as holons (holon-firms). The scenario concerns $I$ independent and geographically dispersed holon-firms, each of them able to produce $K$ different product families, after an appropriate reconfiguration. Assuming that the total planning horizon $\mathrm{T}$ is divided into $N$ sub-periods $t(t=1-N)$; at the beginning of each sub-period the holon-firms make their capacity allocation plan, after having collected backlog and forecast orders according to the capacity they hold and to the demand they have to supply. If the holon-firm capacity is not enough to supply the demand orders, then they can negotiate a portion of capacity with holon-firms whose production capacity exceeds their actual demand. This process leads to a sharing process in which each holon-firm tries to maximize its own profit. By doing so, more value-added collaborations are generated without reducing the individual benefits of the holon-firm. Specifically, each holon-firm $(f)$ computes its accomplishment capacity index (ACI) $S_{f, A C I}$ by measuring the difference between its own workload $(W L)$ and its capacity $(C)$ :

$$
S_{f, A C I}=W L_{f}-C_{f}
$$

Subsequently, holon-firms are classified in overloaded holon-firms (OF), i.e., holons with $S_{f, A C I}>0$, or underloaded holon-firms (UF), i.e., holons with $S_{f, A C I}<0$. Afterwards, holons belonging to OF or UF compute respectively their required capacity $\left(\mathrm{RC}_{f}\right)$ or the capacity they can offer $\left(\mathrm{OC}_{f}\right)$ :

$$
\begin{gathered}
R C_{f}=W L_{f}-C_{f} \\
O C_{f}=C_{f}-W L_{f}
\end{gathered}
$$
[14].

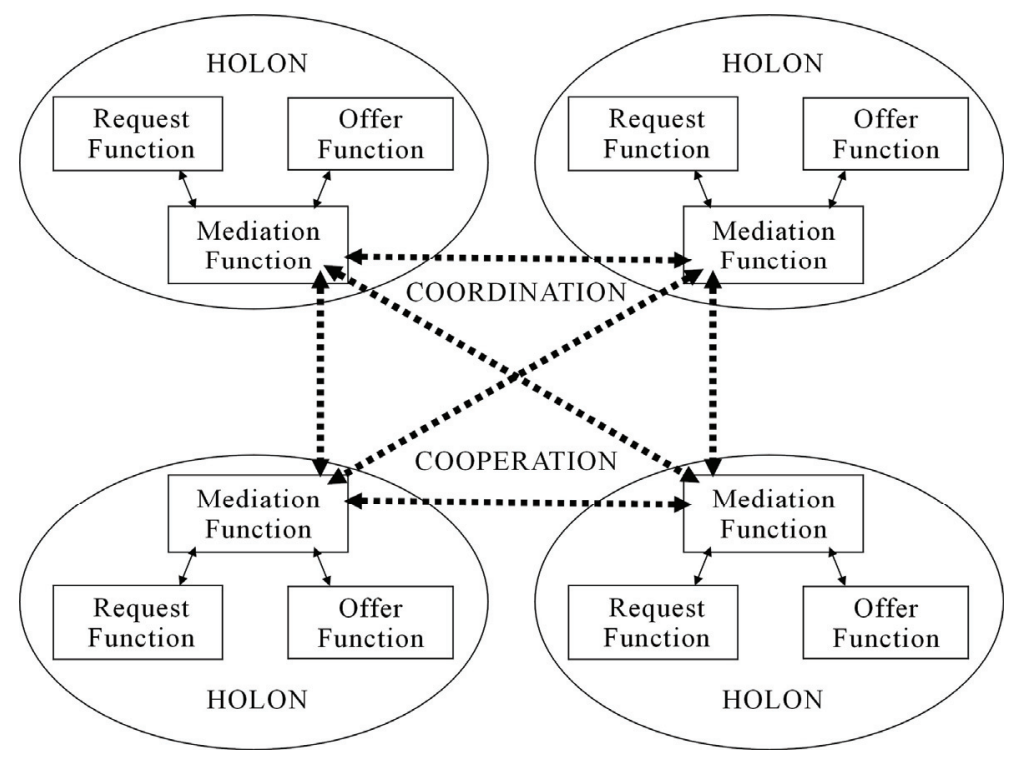

Figure 1. Simplified architecture of a holonic system. 
As showed in Figure 1, each holon operates by using a firm agent with a specific function. The mediator function performs the interaction activities among all the holons: the mediation aims to share the capacity in order to maximize the overall profit of the holonic network. Each holon has the following local knowledge:

- Cik: i-th holon-firm's capacity to produce the k-th product;

- Costik: i-th holon-firm's total production costs to produce the k-th product;

- Priceik: i-th holon-firm's unit product price to sell in k-th holon-firm market ;

- WLik: i-th holon-firm's workload regarding the k-th product;

- Value of OCf or RCf.

\subsection{Multi Agent Architecture and Negotiation Activity}

We adopt the following multi agent architecture to ana- lyze the HPS negotiation's capacities:

- each holon-firm belonging to OF is represented by an OCf Function (OCF) which is in charge for negotiating the capacity with the holon-firms requiring it (RCFs);

- a mediator function (MF) allows communication and coordination between capacities of supplier and applicant holon-firms;

- the negotiation process and interaction workflow between supplier and requester holon-firms is represented by the UML activity diagram in Figure 2 .

The UML diagram is divided in three swim lines, corresponding to the requester, supplier and mediator functions. The first activity is performed by the MF that collects RCF capacities requests, sorted by decreasing amount of capacity. Starting from the higher capacity request, the MF transmits the proposals to OCF holonsfirms in terms of capacities and prices of each quantity. The OCF evaluates requester proposals, in terms of quan-

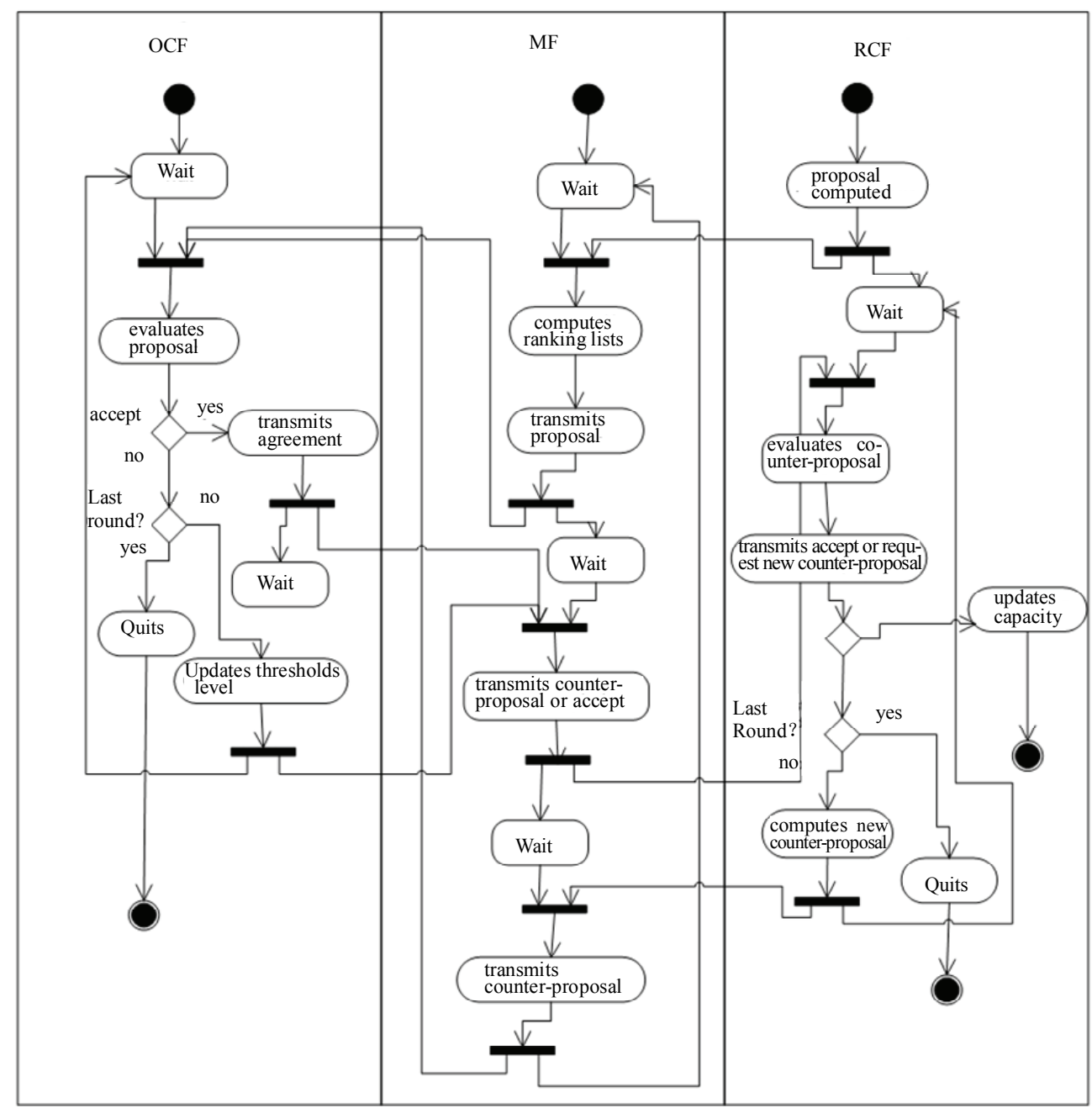

Figure 2. Negotiation approach: UML activity diagram. 
tity and prices, by using the firm's utility functions. OCFs can decide: to quit the negotiation, to accept the proposal or to requests for a new counterproposal when the current step of negotiation is lower than the established maximum one. If the proposal is accepted the agreement is reached, otherwise the RCF evaluates the counter-proposal by using its own utility function. Therefore, the negotiation procedure can be interpreted as a simple auction based negotiation protocol. It defines the environmental relations of the autonomous firms involved in the holonic network, but no indication about the holon-firms decision making behavior (utility function) is given. This means that the negotiation protocol can be adapted to different productive functions.

The HPS collaborative network model adopted is based on a negotiation approach whilst a centralised allocation mechanism is used as benchmark.

The negotiation process is characterized by the following constraints:

- the negotiation is multi-lateral and involves one to many holon-firms;

- the negotiation is an iterative process with a maximum number of rounds, rmax, after that an agreement is reached or the negotiation fails;

- during each round (r), the OCF can submit a new counter-proposal $(\mathrm{N})$ to the RCF while, at $\mathrm{r}=\operatorname{rmax}$, it can only accept or reject;

- the agreement is reached only if the RCF accepts the OCF counter proposal at round $\mathrm{r}<\mathrm{rmax}$; a contract of production collaboration between the supplier and applicant holon-firms follows the agreement; if there are multiple agreements, the first OCF that satisfies the RCF sings the agreement.

- the holon-firms' behavior is assumed to be rational according to its utility function (as defined later);

- the RCF and the OCF are mutually unaware of each others utility function.

The OCF activities can be described as follows:

- Wait: the agent is in an initial state of waiting for a proposal (from RCF);

- Evaluates proposal: the OCF evaluates the proposal of the RCF in terms of required capacity and offered price. At the first round the OCF, communicates the amount of capacity it is willing to offer (the minimum value between the one requested by the RCF and its own unused capacity). Subsequently, the OCF communicates to the RCF if it accepts or refuses the proposed price to exchange the promise amount of capacity. Then the OCF evaluates the proposal of the RCF on the basis of a threshold function given by (4):

val $_{j}^{k, r}=\left[\right.$ price $_{j}^{k}-\left(\right.$ price $\left.\left._{j}^{k}-\cos t_{j}^{k}\right) \cdot \frac{r-1}{r_{\max }-1}\right] \cdot M_{i j}^{k}$

$$
\text { being } M_{i j}^{k}=\min \left(R C_{i}^{k}, O C_{j}^{k}\right)
$$

Expression (4) computed by the OCF is a threshold level. Starting from the market price value, during the negotiation the variable $r$ increments and the threshold level decrease until the value of production costs. In this case the generated profit is null.

At this point the value of the offer is checked according to the following expression:

$$
v a l_{i}^{k, r} \geq v a l_{j}^{k, r}
$$

If (6) is verified, the $j$ th holon-firm supplies the requested capacity of the $i t h$ holon-firm, in this way they reach an agreement and each of them can update their available capacity.

- Threshold level updates: when the OCF refuses the price submitted by the RCF, it updates the threshold level for the next round of negotiation (increases the value of $r$ in expression (4)); if the algorithm reached the last round of negotiation, the OCF simply quits the negotiation.

- Capacity updates: when the negotiation reaches an agreement, the OCF updates the capacity owned. If no more capacity resources are available, OCF quits, otherwise it goes in Wait state.

The activities performed by RCA can be described as follows:

- Proposal elaboration: the RCF elaborates a proposal in terms of price and amount of capacity to acquire, and transmits this information to the MF. The submitted price is obtained by the following expression:

val $_{i}^{k, r}=\left[\right.$ price $_{i}^{k}-\left(\right.$ price $\left.\left._{i}^{k}-\cos t_{i}^{k}\right) \cdot \frac{r_{\max }-r}{r_{\max }-1}\right] \cdot M_{i j}^{k}(7)$

Expression (7) is computed by the RCF and starts with a price equal to production costs, where the generated profit is the same obtained when products are produced by its own holon-firm. During the negotiation, the price is increased until the market price. In this case, the generated profit is null.

- Wait: the RCF waits for counter-proposal by the OCF.

- Counter-proposal computation: if the OCF refuses the proposal and the negotiation is still running, the RCF computes a new counter-proposal (increasing the value of $r$ in expression (7)). Otherwise (i.e., it is the last round of negotiation), the process ends with no agreement.

- Capacity updates: if the negotiation reaches an agreement, the RCF updates its information; if the acquired capacity is exactly equal to the required one, it quits, otherwise it computes a new proposal 
for the residual capacity needed.

The MF coordination activities between OCF and RCF can be described as follows:

- Wait: the MF is in its initial state of waiting for a proposal (from the RCF).

- Computation of ranking list: the MF computes a ranking list among all the holon-firms that requested capacity. The way it does it depends on several variables; in our simulation we consider that the ranking is done favoring holon-firms with high need of capacity to allow them to better satisfy customers' requests.

- Proposal transmition: the MF transmits the proposal computed by RCF to the ranking list of OCF.

- Wait: the MF is waiting for the counter-proposal by all the OCF.

- Counter-proposal transmition: the MF transmits the counter-proposal of the OCF to the RCF.

After all the necessary values have been uploaded, the holon-firm that does not reach the entire capacity it needs is inserted in the ranking list again, and the negotiation starts a new round. To avoid a deadlock, the holon-firm that does not reach any agreement at the end of the negotiation process is removed from the ranking list.

\subsection{Centralised Approach (Benchmark)}

In order to test the benefits of the adopted negotiation approach for the HPS, we develop a centralized model and a Mixed Integer Program (MIP). The objective function is the total profit maximization of the holonic network as a whole:

$$
\max \text { Profit }=\sum_{k} \sum_{i} \sum_{j}\left(\text { Price }_{k}^{i}-C \text { os } t_{k}^{i}\right) * x_{i j}
$$

being $x_{i j}$ the amount of capacity of the ith holon-firm transacted with the $j t h$ holon-firm. Data knowledge of the model is constituted by:

- Price $_{k}^{i}$;

- $\operatorname{Cost}_{k}^{i}$;

- oCf;

- $R C f$,

subject to the following constraints:

$$
\begin{aligned}
& \sum_{i} x_{i j}<=R C_{f}^{j}, \text { for each } j \\
& \sum_{j} x_{i j}<=O C_{f}^{i}, \text { for each } i
\end{aligned}
$$

Constraint (9) forces the whole capacity transaction toward the $j$ th holon-firm to become lower than that requested by the same holon-firm. Constraint (10) forces the whole capacity transaction toward the ith holon-firm to be lower than that offered by the same holon-firm.

\section{Simulation Environment}

The distributed network has been implemented and tested through a simulation environment developed by using the Java Development Kit (JDK) package. The adopted modeling formalism is a collection of independent agents interacting via a discrete events mechanism. The simulation consists of a group of several interacting agents, while simulation proceeds through alternative timetables of discrete events. The considered schedules are data structures that combine actions in the planned order. Two kinds of packages have been developed: the holon-firms' network and the statistical package.

The holon-firms' network package consists of three different agents with specific tasks:

- the holon's agent, supervises local holon-firm data and algorithms;

- the mediator agent, implements the negotiation mechanism among all the holon-firm agents involved in the negotiation;

- the scheduling agent, manages the discrete events that determine the simulation runs.

The statistical package collects the data generated at the end of each simulation run and generates both reports and statistical analyses. The test environment considers the following inputs:

- holon-firms' capacity: the capacity assigned to the holon-firm at the beginning of each sub-period;

- capacity demand: the product demand that the holon-firm tries to satisfy;

- reconfiguration cost: the cost of the holon-firm to change the production from one typology product to another.

- number of product-type: the number of lines of products requested by the market.

The simulations were conducted considering: a number (I) of six of holon-firms $(\mathrm{I}=6)$ and a number $(\mathrm{N})$ of twelve sub-periods $(\mathrm{N}=12)$. At the beginning of each sub-period, the capacity re-allocation process is activated. Input data were randomly generated, being randomly drawn from different distributions as reported in Table 1.

Low and High are referred to the standard deviation's size of the normal distributions. The target profit value is fixed for all the considered holon-firms. The simulation has been conducted for different numbers of producttypes $(\mathrm{k}): \mathrm{k}=1, \mathrm{k}=5$ and $\mathrm{k}=10$, in order to investigate the behavior of the network when the number of pro-

Table 1. Input data.

\begin{tabular}{lcc}
\hline & Low & High \\
\hline Holon-firms' capacity & $\mathrm{N}(100,10 \%)$ & $\mathrm{N}(100,50 \%)$ \\
Capacity demand & $\mathrm{N}(100,10 \%)$ & $\mathrm{N}(100,50 \%)$ \\
Reconfiguration costs & $\mathrm{N}(2,10 \%)$ & $\mathrm{N}(2,50 \%)$ \\
\hline
\end{tabular}


ducts typologies change. The capacity costs are 10 units, therefore the holon-firm reconfiguration costs are considered as $20 \%$ of the capacity costs. The holon-firms have the same mark-up target: $20 \%$. For each simulation run, the goal was to evaluate the unallocated capacity (UC) and the total amount of profit generated by the holon-firms. Simulation experiments were conducted to evaluate, for each run, the measures of performance with a confidence interval of $95 \%$. Combining different levels of all four parameters, 24 simulation classes of experiments were obtained as described in Table 2.

The following performance measures have been considered:

- the total profit (profit); given by the profits generated by all the holon-firms belonging to the network;

- the unallocated capacity (UC); given by the sum of the holon-firms' unallocated capacities.

\section{Simulation Results}

Tables 3-6 report, in percentage, the difference between the performances, evaluated using the proposed distributed coordination model in different environmental conditions. In particular, Table 3 reports the results of the

Table 2. Experimental classes.

\begin{tabular}{|c|c|c|c|c|}
\hline $\begin{array}{l}\text { Experi- } \\
\text { ment } \\
\text { classes }\end{array}$ & $\begin{array}{l}\text { Holon-firm } \\
\text { capacity } C_{p}^{k}\end{array}$ & $\begin{array}{l}\text { Customer } \\
\text { demand } \\
R_{p}^{k}\end{array}$ & $\begin{array}{l}\text { Reconfigura- } \\
\text { tions costs }\end{array}$ & $\begin{array}{l}\text { Product- } \\
\text { type } K\end{array}$ \\
\hline 1 & $\mathrm{~L}$ & $\mathrm{~L}$ & $\mathrm{~L}$ & 1 \\
\hline 2 & $\mathrm{~L}$ & $\mathrm{~L}$ & $\mathrm{H}$ & 1 \\
\hline 3 & $\mathrm{~L}$ & $\mathrm{H}$ & $\mathrm{L}$ & 1 \\
\hline 4 & $\mathrm{~L}$ & $\mathrm{H}$ & $\mathrm{H}$ & 1 \\
\hline 5 & $\mathrm{H}$ & $\mathrm{L}$ & $\mathrm{L}$ & 1 \\
\hline 6 & $\mathrm{H}$ & $\mathrm{L}$ & $\mathrm{H}$ & 1 \\
\hline 7 & $\mathrm{H}$ & $\mathrm{H}$ & $\mathrm{L}$ & 1 \\
\hline 8 & $\mathrm{H}$ & $\mathrm{H}$ & $\mathrm{H}$ & 1 \\
\hline 9 & $\mathrm{~L}$ & $\mathrm{~L}$ & $\mathrm{~L}$ & 5 \\
\hline 10 & $\mathrm{~L}$ & $\mathrm{~L}$ & $\mathrm{H}$ & 5 \\
\hline 11 & $\mathrm{~L}$ & $\mathrm{H}$ & $\mathrm{L}$ & 5 \\
\hline 12 & $\mathrm{~L}$ & $\mathrm{H}$ & $\mathrm{H}$ & 5 \\
\hline 13 & $\mathrm{H}$ & $\mathrm{L}$ & $\mathrm{L}$ & 5 \\
\hline 14 & $\mathrm{H}$ & $\mathrm{L}$ & $\mathrm{H}$ & 5 \\
\hline 15 & $\mathrm{H}$ & $\mathrm{H}$ & $\mathrm{L}$ & 5 \\
\hline 16 & $\mathrm{H}$ & $\mathrm{H}$ & $\mathrm{H}$ & 5 \\
\hline 17 & $\mathrm{~L}$ & $\mathrm{~L}$ & $\mathrm{~L}$ & 10 \\
\hline 18 & $\mathrm{~L}$ & $\mathrm{~L}$ & $\mathrm{H}$ & 10 \\
\hline 19 & $\mathrm{~L}$ & $\mathrm{H}$ & $\mathrm{L}$ & 10 \\
\hline 20 & $\mathrm{~L}$ & $\mathrm{H}$ & $\mathrm{H}$ & 10 \\
\hline 21 & $\mathrm{H}$ & $\mathrm{L}$ & $\mathrm{L}$ & 10 \\
\hline 22 & $\mathrm{H}$ & $\mathrm{L}$ & $\mathrm{H}$ & 10 \\
\hline 23 & $\mathrm{H}$ & $\mathrm{H}$ & $\mathrm{L}$ & 10 \\
\hline 24 & $\mathrm{H}$ & $\mathrm{H}$ & $\mathrm{H}$ & 10 \\
\hline
\end{tabular}

performance measured considering different degrees demand variability.

Demand variability leads to a significant increase of the unsatisfied demand, while the profit of the network shows a small decrease. Table 4 reports the simulation results when considering the distribution of the different capacities possessed by the holon-firms in the network.

The distribution of capacity among the holon-firms combined with network variability leads to high levels of unsatisfied demand, while the total profit of the network shows a low variability. However, the influence on the performances of the network engendered by the holonfirms' capacity variability is not as strong as that caused by customer demand variability.

Table 5 reports the simulation results considering the distribution of reconfiguration costs among holon-firms. The results of the simulation show that the distribution of reconfiguration costs has a very low effect on performance measures.

Finally, the number of product types has been investigated in order to understand how diversification affects the performance of the system. Table 6 reports the in-

Table 3. Simulation results - customer demand.

\begin{tabular}{ccc}
\hline Customer demand & Demand unsatisfied & Total profit \\
\hline Low variability & 402.4 & 13523.49 \\
High variability & 794.08 & 12855.83 \\
Difference & $97.34 \%$ & $-4.94 \%$ \\
\hline
\end{tabular}

Table 4. Simulation results - holon-firm's capacity.

\begin{tabular}{ccc}
\hline Holon-firms' capacity & Demand unsatisfied & Total profit \\
\hline Low variability & 427.4 & 13528.98 \\
High variability & 769.08 & 12850.34 \\
Difference & $79.94 \%$ & $-5.02 \%$
\end{tabular}

Table 5. Simulation results - reconfigurations costs.

\begin{tabular}{ccc}
\hline Reconfiguration costs & Demand unsatisfied & Total profit \\
\hline Low variability & 584.53 & 13218.55 \\
High variability & 583.02 & 13218.55 \\
Percentage difference & $-0.26 \%$ & $0.00 \%$ \\
\hline
\end{tabular}

Table 6. Simulation results - products typology.

\begin{tabular}{ccc}
\hline Number of product typology K & Demand unsatisfied & Total profit \\
\hline 1 & 511.45 & 13362.2 \\
5 & 616.81 & 13158.84 \\
10 & 623.07 & 13134.21 \\
Percentage difference 5-1 & $20.60 \%$ & $-1.52 \%$ \\
Percentage difference 10-1 & $21.82 \%$ & $-1.71 \%$ \\
Percentage difference 10-5 & $1.01 \%$ & $-0.19 \%$ \\
\hline
\end{tabular}



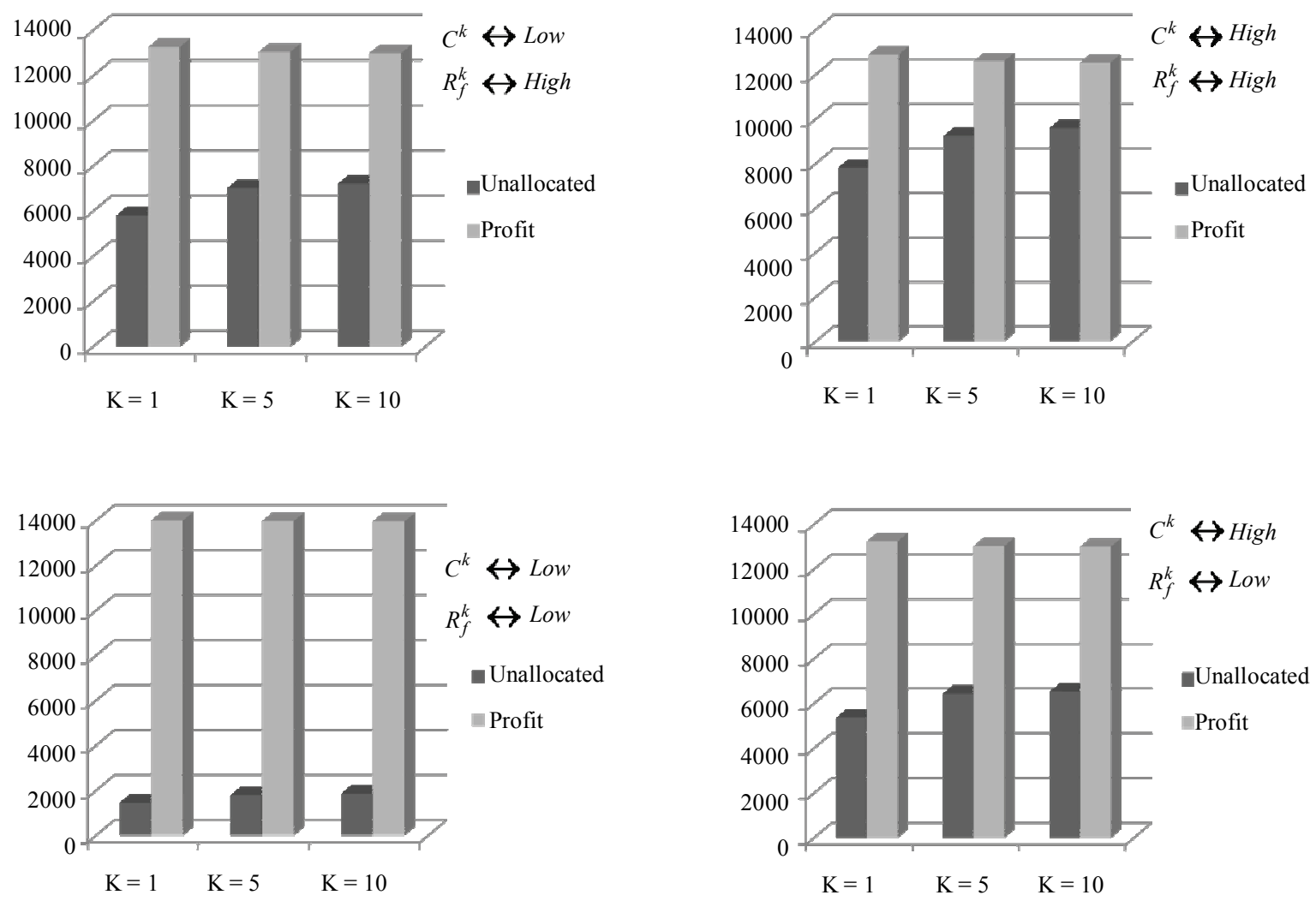

Figure 3. HPS responsiveness to demand and capacity variability: Bar charts represent unallocated capacity and whole HPS profit at different $\mathrm{K}$ diversification levels.

fluence of the number of products types $k$ on the system performance indicators, here calculated as the mean of the customer demand and holon-firms capacity variability. The simulation shows that, while the unsatisfied demand grows as the number of products types increases, the effect on the network's profit reduction is attenuated. As a consequence of product differentiation, the responsiveness of HPS to market turbulence is able to alleviate profit variations. Moreover, the difference in percentage obtained among one and five product types is close to the difference obtained with one to ten types. No relevant percentage difference exists among the result with five to ten products types. This last result highlights that, in a six holons production system, the greatest profit make up takes place with a products differentiation lower than five typologies, and that product differentiation is a key factor of adaptability to environment evolutions.

Figure 3 shows the bar charts, for each class of experiments, representing the holonic system performance variations due to variations of $k$ (differentiation), measured by the HPS unallocated capacity and profit. The four diagrams highlight that unallocated capacity raises with $k$, whereas the degree of profit reduction is de- creased. This counterintuitive result draws attention to the mechanism of profit reduction attenuation, which is typical of the holonic system. In fact, the negotiation protocol combined with the holonic mediator function, guarantees an optimal capacity allocation and distribution. While negotiated capacities are transacted at a lower marginal profit, they still generate a supplementary profit for the HPS as a whole.

Although the positive effects of the collaborative negotiation approach is clear for the holonic network performance as a whole, the sustainability of the HPS depends on the wealth of the single holons, upon which the network structure relies. Further research development could concern the analysis of the study of single holonfirm profit dynamics, in order to understand the sustainability of the multi-agent negotiation approach applied to HPS in greater depth.

\section{Conclusions}

This paper proposes a cooperative approach among holon-firms that operate in the same heterachical network as holarchy. Cooperation is considered in terms of trad- 
ing capacity and the holon-firms can be sellers or buyers, depending on the situation. The methods used to supportthe holon-firms network are: a multi-agent system (MAS) to implement the distributed architecture and a negotiation approach to develop the cooperation mechanism.

In this paper we use a simulation environment developed in Java language, in order to test the proposed approach. The performance measures considered have been network profit and unsatisfied demand. These performances have been evaluated in several environmental conditions: different predictability of customer demand, diverse holon-firms' capacity and reconfiguration costs variability in the network. The simulation results highlighted the added value produced by the cooperative approach of the Holonic Production System (HPS), especially in turbulent or variable environmental conditions. Specifically:

- the cooperative approach proposed is robust, in fact the total profit of the network keeps the same level in the different conditions tested;

- the variability of reconfiguration costs among the holon-firms has very low effects on the performance measured;

- the increase of the other three parameters (demand variability, holon-firms' capacity variability and number of product types) leads to an increase in the level of unsatisfied demand;

- the variables that influence HPSs' performance are, in decreasing order: demand variability, variability of holon-firm capacity and number of product types.

The analysis conducted highlighted that the adoption of a cooperative approach leads to superior performance on behalf of the holonic network when the environment is more dynamic. We call this effect network resilience to market turbulence threats.

Further developments of the research proposed could concern: a dynamic investigation of the evolution of the participants in the network, considering also the effect of the growing number of holon firms and product diversification in the HPS; an analysis of the effects of the environmental unpredictability on the performance of the single holon firms, in terms of demand satisfaction and profit, as a complement to the system level analysis proposed in this paper; furthermore, an extension of the aforementioned study would consent to improve the basic understanding of the local holon sustainability versus the global network welfare.

The simulation environment has been entirely developed by using an open source code with an object oriented architecture (Java), so that the multi agent framework developed for our research purposes can be easily upgraded to build a real digital managerial system for real holon-firm networks. This choice allows to reduce investment time and risk of building a real system able to manage networked holon-firms like a HPS.

\section{REFERENCES}

[1] G. Dominici, "Demand Driven Supply Chain ed Innovazione: Il Sistema Logistico-Produttivo per la Soddisfazione dei Bisogni del Cliente,” In: A. Purpura and G. Fazio, Eds., Economia e Gestione Dell'innovazione nelle PMI, FrancoAngeli, Milan, 2008.

[2] G. Dominici, "Holonic Production System to Obtain Flexibility for Customer Satisfaction," Journal of Service Science \& Management, Vol. 1, No. 3, 2008, pp. 251-254.

[3] F. Frederix, "From Production to a Product Perspective. New Industrial Scenario?” In: S. Yoon, et al., Evolution of Supply Chain Management, Symbiosis of Adaptive Value Networks and ICT, Kluwer Academic Publishers, Norwell, 2004.

[4] L. Gou, P. B. Luh and Y. Kyoya, "Holonic Manufacturing Scheduling: Architecture, Cooperation Mechanism, and Implementation," Computers in Industry, Vol. 37, No. 3, 1998, pp. 213-231.

[5] J. Hatvany, "Intelligence and Cooperation in Heterarchic Manufacturing Systems," Robotics \& Computer-Integrated Manufacturing, Vol. 2, No. 2, 1985, pp. 101-104.

[6] H. van Brussel, L. Bongaerts, J. Wyns, P. Valckenaers and T. van Ginderachter, "A Conceptual Framework for Holonic Manufacturing: Identification of Manufacturing Holons," Journal of Manufacturing Systems, Vol. 18, No. 1, 1999, pp. 35-52.

[7] P. Valckenaers, E. Bonneville, H. van Brussel, L. Bongaerts and J. Wyns, "Results of the Holonic Control System Benchmark at the KULeven," Proceedings of Computer Integrated Manufacturing and Automation Technology Conference, Troy, New York, 1994, pp. 128-133.

[8] T. Moyaux, B. Chaib-draa and S. D'Amours, "Supply Chain Management and Multiagent Systems: An Overview," In: B. Chaib-draa and J. P. Müller, Eds., Multiagent Based Supply Chain Management, Springer, Ontario, 2006.

[9] M. Paolucci and R. Sacile, "Agent-Based Manufacturing and Control Systems," CRC Press, Boca Raton, 2005.

[10] G. M. Golinelli, "Viable System Approach (VSA)," Community Economic Development Association of Michigan, Michigan, 2010.

[11] A. Koestler, "The Ghost in the Machine," Arkana, London, 1967.

[12] C. Saccani, "Il Sistema Olonico," Sistemi \& Impresa, Vol. 2, 1996, pp. 29-45.

[13] M. A. Arbib, "The Handbook of Brain Theory and Neural Networks," MIT Press, Boston, 1995.

[14] P. Renna and P. Argoneto, "Capacity Allocation in MultiSite Environment: A Multi Agent Systems Approach,” In: Proceedings of Intelligent Computation in Manufacturing Engineering, Naples, 2008. 